\title{
G s.mastace \\ The Change of GFAP or S100B Concentration in Serum Before and After Carotid Artery Stenting
}

\section{Xiaofan Yuan}

Sichuan Academy of Medical Sciences and Sichuan People's Hospital https://orcid.org/0000-00028789-9623

\section{Shu Yang}

Sichuan Academy of Medical Sciences and Sichuan People's Hospital

\section{Duozi Wang}

Sichuan Academy of Medical Sciences and Sichuan People's Hospital

\section{Lei Guo}

Sichuan Academy of Medical Sciences and Sichuan People's Hospital

\section{Jianhong Wang}

Sichuan Academy of Medical Sciences and Sichuan People's Hospital

\section{Fuqiang Guo ( $\sim$ guofuqiang2005@126.com )}

Sichuan Academy of Medical Sciences and Sichuan People's Hospital https://orcid.org/0000-00020665-4414

\section{Research}

Keywords: Glial fibrillary acidic protein, S100B Protein, Carotid Artery Stenting, Digital Subtraction Angiography, Evaluation, Monitor, cerebral biochemical marker

Posted Date: December 3rd, 2019

DOl: https://doi.org/10.21203/rs.2.18007/v1

License: (c) (1) This work is licensed under a Creative Commons Attribution 4.0 International License. Read Full License 


\section{Abstract}

In this study we monitored the concentration change of the cerebral biomarkers Glial fibrillary acidic protein (GFAP) and S100B Protein (S100B) in serum of the patients, who were performed with carotid artery stenosis before and after carotid artery stent implantation. In total, thirty patients with unilateral carotid stenosis who underwent carotid artery stenting and had no complications were enrolled in the carotid artery stenting (CAS) group. The blood samples of operation patients were collected on three different time points: T1: the day before operation; T2: one day after operation (24 hours); T3, three days after operation (72 hours). Thirty patients who were excluded for carotid artery stenosis by Digital Subtraction Angiography (DSA) were selected as the control group. The blood samples of patients in control group were collected at D1 (before DSA) and D2 (24 hours after DSA). The concentrations of GFAP and S100B in serum from patients of both groups were measured with ELISA. The results showed that the serum concentrations of GFAP and S100B in control group did not change significantly before and after DSA ( $P>0.05)$; while the serum concentrations of GFAP and S100B was higher in the CAS group before operation than those in DSA group $\mathbb{P P}<0.05 \otimes$. In the operation group, GFAP and S100B increased significantly on the first day after operation (T2) and decreased gradually on the third day after operation (T3) but increased compared with that before operation (T1) with statistical significance $(P<0.05)$. For patients with symptomatic stenosis before operation, the concentrations of GFAP and S100B in serum were higher than those in patients without symptomatic stenosis, and there was statistical difference between them $(P<0.05)$, but there was no statistical significance between them after operation $(P>$ 0.05). So we confirm the cerebral biochemical markers GFAP and S100B have a certain change trend after CAS, which can be used as the cerebral biochemical markers in the pre-operative evaluation and post-operative efficacy in monitoring of carotid stent implantation.

\section{Introduction}

Carotid Artery Stenting (CAS) has been the micro-surgical method advocated in the recent 20 years. Among patients with symptomatic or asymptomatic carotid stenosis, CAS and Carotid Endarterectomy (CEA) are preventive measures for ischemic cerebrovascular disease. The risk of the composite primary outcome of stroke, myocardial infarction, and death do not significantly differ in patient groups undergoing CAS or undergoing CEA [1]. However, CAS is easy to accept because of its minimally invasive operation and short postoperative hospitalization period [2].

In clinical work, the long-term effect monitoring of CAS patients can evaluate the improvement of postoperative stenosis by Digital Subtraction Angiography (DSA), Computed Tomography Angiography (CTA), Magnetic Resonance Angiography (MRA) and other imaging methods. On the one hand, it is not accurate to use only the diameter of vasodilation to express the effective perfusion after operation. On the other hand, for the short-term effect evaluation after CAS, the above imaging examination methods have the disadvantages of vulnerability to human body, poor repeatability, high cost and so on. 
Cerebral biochemical markers are the research hotspots in the recent 20 years, mainly including GFAP, S100B, Neuron Specific Enolase (NSE) and Myelin Basic Protein (MBP), etc [3]. At present, it has been confirmed that the concentrations of GFAP, S100B, NSE, MBP in cerebrospinal fluid and serum are related to the size of cerebral infarction area, the deficit of neurological function and the pre- and post- recovery of function [3-6]. So the researchers advocate the use of cerebral biochemical markers in Cerebrospinal Fluid (CSF) and serum combined with imaging examination as an assisting tool for the diagnosis, disease assessment and prognosis monitoring of cerebral infarction or other brain injury.

Therefore, this study combined CAS and cerebral biochemical markers to explore the expression of GFAP and S100B before and after CAS. Although there are imaging tools as the examination methods for the improvement of the degree of vascular stenosis after CAS operation, this is the first time for cerebral biochemical markers as the evaluation method.

\section{Methods}

Research object: From April 2018 to August 2019, DSA was performed in the Department of Neurology of Sichuan Provincial People's Hospital to diagnose unilateral carotid stenosis, then patients underwent carotid stent implantation were assigned as the operation group. The inclusion criteria of the operation group were: (1) symptomatic stenosis $\geq 50 \%$ and the clinical symptoms were consistent with the vascular area of stenosis; (2) asymptomatic stenosis $\geq 80 \%$; (3) age > 18 years. Exclusion criteria of the operation group: (1) patients with brain injury within 6 months; (2) patients with nervous system related tumor diseases; (3) patients with infectious diseases of nervous system (such as encephalitis, meningitis, myelitis, etc.); (4) patients with demyelination and degenerative diseases of nervous system (such as multiple sclerosis, optic neuromyelitis, Parkinson's disease, Alzheimer's disease, etc.); (5) patients with severe heart, liver, kidney Lung disease could not tolerate surgery. At the same time, the patients who were excluded from intracranial and extracranial vascular stenosis by DSA in neurology department were selected as the control group.

Surgical procedure: The patients in the operation group were given dual antiplatelet therapy (aspirin $100 \mathrm{mg}$ and clopidogrel $75 \mathrm{mg}$ ) three days before operation. The operation was performed by more than two experienced neuroscientists. Under the guidance of the guiding wire, the finger guide tube was placed near the opening of the beginning of the internal carotid artery in the affected side, and the protective umbrella was placed at the distal end of the stenosis artery. Then the stent was placed along the guide wire in the stenosis area. After precise positioning, the stent was implanted. The operation was completed, after the angiography showed that the carotid artery was unobstructed, the stent was well attached to the wall, no obvious residual stenosis was found, and the anterior blood flow was normal (TICI Level 3) [7]. Aspirin was taken for life after operation, combined with clopidogrel for at least 6 months.

Evaluation of carotid stenosis: The degree of carotid stenosis was determined by more than two neurologists, and the criteria were calculated according to the European Carotid Surgery Study (ECST) [8] 
and North American symptomatic carotid endarterectomy (NASCET) [9]. That is, stenosis degree $(\%)=(1-$ diameter of stenosis / diameter of distal normal lumen) X100\%. The classification included: normal / mild stenosis $(<50 \%)$, moderate stenosis $(50-69 \%)$, severe stenosis $(70-99 \%)$, complete occlusion (100\%).

Collection and measurement on serum samples: In the operation group, $3 \mathrm{ml}$ of blood was collected in the morning before operation ( $\mathrm{T} 1$ ), one day after operation (T2) and three days after operation (T3). In the control group, the blood was collected in the morning before DSA (D1) and one day after operation (D2). The blood samples were stored at $-80^{\circ} \mathrm{C}$ before measurement. The ELASA procedure for detecting concentrations of serum GFAP and S100B was as follows: either anti-GFAP (Human GFAP ELISA KIT, ZC34594) or anti-S100B (Human S100B ELISA KIT, ZC-32056) antibodies were coated in 96 well microporous plates to make solid-phase carriers, and standards or samples are added to the micropores respectively. The GFAP and S100B are attached to their specific antibody binding on the solid-phase carriers, then after thorough washing, GFAP and S100B antibodies are added. After the unconjugated biotin antibodies are cleaned, HRP labeled avidin is added, and then they are washed thoroughly again. TMB substrate was added and converted to blue under the catalysis of peroxidase, and finally to yellow under the action of acid. The color depth was positively correlated with concentration of either GFAP or $\mathrm{S} 100 \mathrm{~B}$ protein. The absorbance (OD value) was measured at $450 \mathrm{~nm}$ wavelength with the microplate reader, and the sample concentrations were calculated.

Statistical analysis: SPSS 22.0 was used for statistical analysis. The measurement data was expressed by mean \pm standard deviation $(X \pm s)$, and $t$ test was used for the comparisons between groups. The counting data was expressed by frequency and percentage, and chi-square test was used for comparison. Statistical significance was defined as $\mathrm{P}<0.05$.

\section{Results}

Demographic data: Following the criteria, 32 patients were enrolled in the operation group. Among them, 2 male patients were excluded because of serious postoperative complications. In total 30 patients, there were 20 males and 10 females. Their ages were 42 to 77 years old with an average age of $69.05 \pm 10.83$ years. There were 30 patients in the control group, 16 males and 14 females, aged 38 to 81 years, with an average age of $67.34 \pm 7.56$ years. There was no statistical difference between the operation group and the control group $(\mathrm{P}>0.05, \mathrm{Table} 1)$.

Image analysis on patients in the operation group: There were 30 patients in the operation group, including 18 patients with symptomatic stenosis and 12 patients with asymptomatic stenosis. There were 10 cases of moderate stenosis, 17 cases of severe stenosis and 3 cases of complete occlusion. The results of cerebral angiography before and after shown in Figure 1A, $1 \mathrm{~B}$.

The changes of serum GFAP and S100B in different groups: The serum concentrations of GFAP and S100B in the operation group and the control group before and after operation are shown in Table 2. (1)The serum GFAP and S100B in the control group had no significant change before and after DSA (P > 0.05). The serum GFAP and S100B in the operation group were higher than those patients in the control group, and the difference was statistically significant (P 
$<0.05)$. The serum GFAP and S100B in the operation group were significantly higher on the first day after operation, and decreased on the third day after operation, but still higher than those before operation. Comparing to prior to operation, the level of both GFAP and S100B were statistically significantly increased at T1 and T3 (P<0.05, Figure 2A and B).

(2)The serum concentrations of GFAP and S100B in patients with symptomatic stenosis before operation were higher than those in patients with asymptomatic stenosis, and the difference there was statistically significant $(\mathrm{P}<0.05)$, but there was no significant difference between both groups after operation ( $P>0.05$, Table 3 and 4 ).

(3)For patients with different degree of carotid artery stenosis, the serum GFAP and S100B levels had no change before and after operation $(\mathrm{P}>0.05)$.

\section{Tables and figures}

Table 1 Demographic data in the DSA and CAS patient groups

\begin{tabular}{c|c|c|c|c|}
\hline Category & DSA group & CAS group & T or $\chi^{2}$ & P value \\
\hline Mean \pm SD age(y) & $67.34 \pm 7.56$ & $69.05 \pm 10.83$ & -0.373 & 0.81 \\
\hline Sex(male) & $16(53.33)$ & $20(66.67)$ & 1.286 & 0.43 \\
\hline Diabetes & $13(43.33)$ & $2(73.33)$ & 0.689 & 0.76 \\
\hline Hypertension & $16(53.34)$ & $21(70.00)$ & 0.694 & 0.63 \\
\hline Hyperlipidemia & $12(40.00)$ & $17(56.67)$ & 0.068 & 0.20 \\
\hline Heart disease & $8(26.67)$ & $9(30.00)$ & 0.424 & 0.34 \\
\hline Current smoker & $5(16.67)$ & $7(23.33)$ & 1.494 & 0.29 \\
\hline Stroke history & $5(16.67)$ & $18(60.00)$ & 3.320 & 0.43 \\
\hline HbA1c (\%) & $5.88 \pm 0.40$ & $6.04 \pm 0.76$ & -2.000 & 0.11 \\
\hline Hyperuricemia & $304.23 \pm 47.30$ & $325.44 \pm 50.68$ & 1.267 & 0.27 \\
\hline Obesity & $12(40.00)$ & $15(50.00)$ & 0.546 & 0.51 \\
\hline
\end{tabular}

Table 2 The serum concentrations of GFAP and S100B in patients at different time points 


\begin{tabular}{c|c|c|}
\hline Group & GFAP $₫ \mathrm{pg} / \mathrm{ml} \square$ & S100B $₫ \mathrm{ng} / \mathrm{ml}$ \\
\hline D1 & $20.059 \pm 10.219$ & $0.853 \pm 0.162$ \\
\hline D2 & $21.392 \pm 09.022$ & $0.909 \pm 0.127$ \\
\hline T1 & $25.392 \pm 11.022$ & $1.500 \pm 0.804$ \\
\hline T2 & $29.877 \pm 14.979$ & $1.974 \pm 1.082$ \\
\hline T3 & $27.038 \pm 12.294$ & $1.786 \pm 0.975$ \\
\hline
\end{tabular}

Table 2: The serum concentrations of GFAP and S100B did not change significantly before and after DSA (D1 vs. D2םPa 0.05). Both GFAP and S100B in the DSA group had lower concentrations when compared with the CAS group aD1/D2<T1/T2/T3ロD1 vs T1凸T1 vs T2 and T1 vs T3ロP<0.05).

Table 3 Serum concentration of GFAP in symptomatic and asymptomatic stenosis patients

\begin{tabular}{|c|c|c|c|}
\hline Group & Symptomatic stenosis & asymptomatic stenosis & P value \\
\hline T1 & $27.685 \pm 10.265$ & $24.960 \pm 10.167 *$ & 0.031 \\
\hline T2 & $30.317 \pm 12.384$ & $28.257 \pm 09.742^{* *}$ & 0.102 \\
\hline T3 & $28.984 \pm 11.309$ & $25.746 \pm 11.473^{* *}$ & 0.298 \\
\hline
\end{tabular}

$* \mathrm{P}<0.05 \square * * \mathrm{P}>0.05$

Table 4 Serum concentration of S100B in symptomatic and asymptomatic stenosis patients

\begin{tabular}{|c|c|c|c|}
\hline Group & Symptomatic stenosis & asymptomatic stenosis & P value \\
\hline T1 & $1.675 \pm 1.212$ & $1.329 \pm 0.881^{*}$ & 0.048 \\
\hline T2 & $2.170 \pm 1.007$ & $1.845 \pm 0.987^{* *}$ & 0.376 \\
\hline T3 & $1.829 \pm 1.102$ & $1.520 \pm 1.003^{* *}$ & 0.769 \\
\hline
\end{tabular}

Table 3-4: Before carotid artery stenting, the serum concentrations of GFAP and S100B in patients with symptomatic carotid stenosis were higher than patients with asymptomatic carotid stenosis $(* \mathrm{P}<0.05)$. However, there was no significant difference in patients with symptomatic or asymptomatic carotid stenosis after operation $\square^{* *} \mathrm{P}>0.05$.

[Figures 1 and 2] 


\section{Discussion}

Studies have confirmed that the rate of symptomatic internal carotid artery stenosis is $>50 \%$, the rate of asymptomatic internal carotid artery stenosis is $>80 \%$, and at least one patient with high risk factors for CEA treatment, CAS efficacy is not inferior to CEA [10]. Three large international multicenter randomized controlled trials also failed to demonstrate that CAS was less effective than CEA [11-13]. In addition, there was no significant difference in primary endpoint (perioperative stroke, myocardial infarction, and ipsilateral stroke of responsible vessels within 4 years) in both symptomatic and asymptomatic carotid stenosis (CAS group $7.2 \% \pm 0.8$ ) $\%$, CEA group $6.8 \% \pm 0.8 \%, P=0.51$ ); but in terms of health-related quality of life, due to the small injury of CAS and short postoperative hospitalization period, it will benefit more in the short term [14].

Astrocytes are the main glial cells in brain tissue, which interact with neuronal cells and function to regulate neurotransmitters, promote immune responses, regulate intracranial blood flow, ions and antioxidants [15]. GFAP and S100B are the main components and signature proteins of astrocytes, and their presence ensures the maintenance and functional function of astrocyte morphological structure [16-17]. The increase of GFAP and S100B in CSF [18-19] and blood [20] reflects the formation of astrocyte filaments in the central nervous system. High concentrations of GFAP and S100B suggest the destruction of acute brain tissue. A moderate increase suggests the astrocytosis, the formation of scars, and delayed ischemic tolerance [21], which plays an important role in promoting neuronal survival and repairing after brain injury [22]. In addition, Herrmann et al found that the release of GFAP and S100B were significantly correlated with the incidence of cerebral infarction. While for patients with lacunar or mild stroke, GFAP was found to be a more sensitive cerebral biochemical marker [23].

The results in this study show that the serum concentration of GFAP and S100B from the operation patients' serum is higher than that in the control group. DSA has confirmed that the patients in the operation group have different degrees of carotid artery stenosis, which makes the brain tissue in the state of chronic ischemia and hypoxia, and chronic cerebral ischemia will inevitably lead to brain tissue damage. Meanwhile, astrocytes are very sensitive to cerebral ischemia and hypoxia, which could cause the astrocytes produce excessive GFAP and S100B into the CSF. These proteins as biomarkers can be in turn released into the peripheral blood through impaired blood-brain barrier, so that the increases of GFAP and $\mathrm{S} 100 \mathrm{~B}$ could be detected in blood serum. In addition, for patients with symptomatic carotid stenosis, the increased level of GFAP and S100B in serum is more significant. After CAS operation, GFAP and S100B in serum increased first at T2 and then decreased at T3, but they both were higher than those before CAS operation. The long-term stenosis of carotid artery can cause brain tissue to establish a certain collateral circulation. When the application of the CAS mechanically expands the blood vessels, the temporary relative balanced brain tissue perfusion established before operation can be ruined, so that the excessive release of GFAP and S100B by astrocytes may happen which could be a response of cerebral ischemia-reperfusion. However, with the release of the stenosis after CAS, the blood flow of the stenosis or occlusion vessels was reestablished, the cerebral ischemia and hypoxia injury caused by insufficient blood perfusion was gradually recovered, which was reflected in the decrease of production of 
both GFAP and S100B proteins locally. It has been confirmed that the moderate increase of GFAP and $\mathrm{S} 100 \mathrm{~B}$ is related to repairing after brain injury [22]. Therefore, the level of biochemical markers within 3 days after operation is still higher than that before operation, suggesting that brain tissue is still in the process of injury repairing after CAS. In addition, it is reported that the reactive gliocytosis has dual effects [23]. When it cannot be solved in the acute and early chronic phase after injury, the reactive gliosis can have negative impact or consequences to injury area. If the intervention measures are adjusted correctly in the best time window, new methods of treating nervous system injury may be developed. The data in our current study is consistent with the results observed by Wunderlich MT [24]. When alteplase was used to a thrombolysis to patients with middle cerebral artery occlusion, GFAP concentration can slightly decrease comparing to those in patients without thrombolysis, but it is still higher than normal control. For patients with different degrees of carotid artery stenosis, the serum concentrations of GFAP and S100B did not change significantly before and after operation. We considered that the cause of this result may be due to the mixed analysis of the patients with symptomatic and asymptomatic stenosis.

For the evaluation of the short-term effect after CAS, the changes of GFAP and S100B in serum can be monitored to timely reflect the changes after operation; for the evaluation before operation and the longterm monitoring of the effect after operation, biochemical markers and imaging tools can be used as the evaluation means; at the same time, the detection of GFAP and S100B in serum has the advantages of simplicity, low price, repeatability and small damage. However, the critical serum values of GFAP and S100B with different degrees of stenosis, different stages of operation and normal human need further study. In addition, the correct intervention of GFAP and S100B in the best time window after carotid stent implantation may lead to the development of new methods for the treatment of nervous system injury.

\section{Abbreviations}

Carotid Artery Stenting (CAS)

Carotid Endarterectomy (CEA)

Digital Subtraction Angiography (DSA)

Cerebrospinal Fluid (CSF)

Computed Tomography Angiography (CTA)

Glial fibrillary acidic protein (GFAP)

Myelin Basic Protein (MBP)

Magnetic Resonance Angiography (MRA)

Neuron Specific Enolase (NSE)

S100B Protein (S100B) 


\section{Declarations}

Ethics approval and consent to participate: This study was reviewed by the ethics committee of Sichuan Provincial People's Hospital, and all patients signed informed consent.

Consent for publication: Not applicable.

Availability of data and materials: The datasets used and/or analysed during the current study are available from the corresponding author on reasonable request.

Competing interests: The authors declare that they have no competing interests.

Funding: This research did not receive any specific grant from funding agencies in the public, commercial, or not-for-profit sectors.

Authors' contributions: Xiaofan Yuan analyzed the patient data and wrote the manuscript. Jianhong Wang and Shu Yang operated the surgery. Duozi Wang collected the basic data of patients. Lei Guo collected the serum and Fuqiang Guo designed the study. All authors read and approved the final manuscript.

Acknowledgements: We would like to thank all the participants in this study, workers of Lilai Biological laboratory for technical support and professor Wang for language instruction.

\section{References}

1. Brott T G, Hobson R W, Howard G, et al. Stenting versus endarterectomy for treatment of carotidartery stenosis [J]. New England Journal of Medicine, 2010, 363(1): 11-23.

2. Saleem T, Baril D T. Carotid Artery Stenting[M]//StatPearls [Internet]. StatPearls Publishing, 2018.

3. Lamers K J B, Vos P, Verbeek M M, et al. Protein S-100B, neuron-specific enolase (NSE), myelin basic protein (MBP) and glial fibrillary acidic protein (GFAP) in cerebrospinal fluid (CSF) and blood of neurological patients[J]. Brain research bulletin, 2003, 61(3): 261-264.

4. Wunderlich $M T$, Ebert $A D, K r a t z ~ T$, et al. Early neurobehavioral outcome after stroke is related to release of neurobiochemical markers of brain damage [J]. Stroke, 1999, 30(6): 1190-1195.

5. Ahmad O, Wardlaw J, Whiteley W N. Correlation of levels of neuronal and glial markers with radiological measures of infarct volume in ischemic stroke: a systematic review [J]. Cerebrovascular diseases, 2012, 33(1): 47-54.

6. Glushakova O Y, Glushakov A V, Miller E R, et al. Biomarkers for acute diagnosis and management of stroke in neurointensive care units [J]. Brain circulation, 2016, 2(1): 28.

7. Zaidat $0 \mathrm{O}$, Yoo A J, Khatri P, et al. Recommendations on angiographic revascularization grading standards for acute ischemic stroke: a consensus statement[J]. Stroke, 2013, 44(9): 2650-2663. 
8. European Carotid Surgery Trialists' Collaborative Group. Randomised trial of endarterectomy for recently symptomatic carotid stenosis: final results of the MRC European Carotid Surgery Trial (ECST)[J]. The Lancet, 1998, 351(9113): 1379-1387.

9. Beneficial effect of carotid endarterectomy in symptomatic patients with high-grade carotid stenosis. North American symptomatic carotid endarterectomy trial collaborators [J]. N Engl J Med, 1991, 325(7): 445-453.

10. Yadav J S, Wholey M H, Kuntz R E, et al. Protected carotid-artery stenting versus endarterectomy in high-risk patients[J]. New England Journal of Medicine, 2004, 351(15): 1493-1501.

11. Eckstein H H, Ringleb P, Allenberg J R, et al. Results of the Stent-Protected Angioplasty versus Carotid Endarterectomy (SPACE) study to treat symptomatic stenoses at 2 years: a multinational, prospective, randomised trial [J]. The Lancet Neurology, 2008, 7(10): 893-902.

12. Eckstein H H, Ringleb P, Allenberg J R, et al. Results of the Stent-Protected Angioplasty versus Carotid Endarterectomy (SPACE) study to treat symptomatic stenoses at 2 years: a multinational, prospective, randomised trial [J]. The Lancet Neurology, 2008, 7(10): 893-902.

13. Mas J L, Trinquart L, Leys D, et al. Endarterectomy Versus Angioplasty in Patients with Symptomatic Severe Carotid Stenosis (EVA-3S) trial: results up to 4 years from a randomised, multicentre trial[J]. The Lancet Neurology, 2008, 7(10): 885-892.

14. Mantese V A, Timaran C H, Chiu D, et al. The carotid revascularization endarterectomy versus stenting trial (CREST) stenting versus carotid endarterectomy for carotid disease [J]. Stroke, 2010, 41: S31-S34.

15. Sun L, Zhang Y, Liu E, et al. The roles of astrocyte in the brain pathologies following ischemic stroke[J]. Brain injury, 2019, 33(6): 712-716.

16. Magaki S D, Williams C K, Vinters H V. Glial function (and dysfunction) in the normal \& ischemic brain [J]. Neuropharmacology, 2018, 134: 218-225.

17. Tateishi N, Shimoda T, Yada N, et al. S100B: astrocyte specific protein [J]. Japanese journal of psychopharmacology, 2006, 26(1): 11-16.

18. Rosengren L E, Wikkels $\varnothing$ C, Hagberg L. A sensitive ELISA for glial fibrillary acidic protein: application in CSF of adults [J]. Journal of neuroscience methods, 1994, 51(2): 197-204.

19. Aurell A, Rosengren L E, Karlsson B, et al. Determination of S-100 and glial fibrillary acidic protein concentrations in cerebrospinal fluid after brain infarction [J]. Stroke, 1991, 22(10): 1254-1258.

20. Schiff L, Hadker N, Weiser S, et al. A literature review of the feasibility of glial fibrillary acidic protein as a biomarker for stroke and traumatic brain injury [J]. Molecular diagnosis \& therapy, 2012, 16(2): 79-92.

21. Hirayama Y, Koizumi S. Astrocytes and ischemic tolerance [J]. Neuroscience research, 2018, 126: 5359.

22. Ye $H$, Wang L, Yang $X K$, et al. Serum $S 100 B$ levels may be associated with cerebral infarction: a meta-analysis [J]. Journal of the neurological sciences, 2015, 348(1-2): 81-88. 
23. Herrmann, P.E. Vos, M.T. Wunderlich, C.H. de Bruijn, K.J.Lamers, Release of glial tissue-specific protein after acute stroke: a comparative analysis of serum concentrations of protein S-100B and glial fibrillary acidic protein, Stroke 31 (2000) 2670-2677.

24. Wunderlich M T, Wallesch $C W$, Goertler M. Release of glial fibrillary acidic protein is related to the neurovascular status in acute ischemic stroke [J]. European journal of Neurology, 2006, 13(10): 11181123.

\section{Figures}

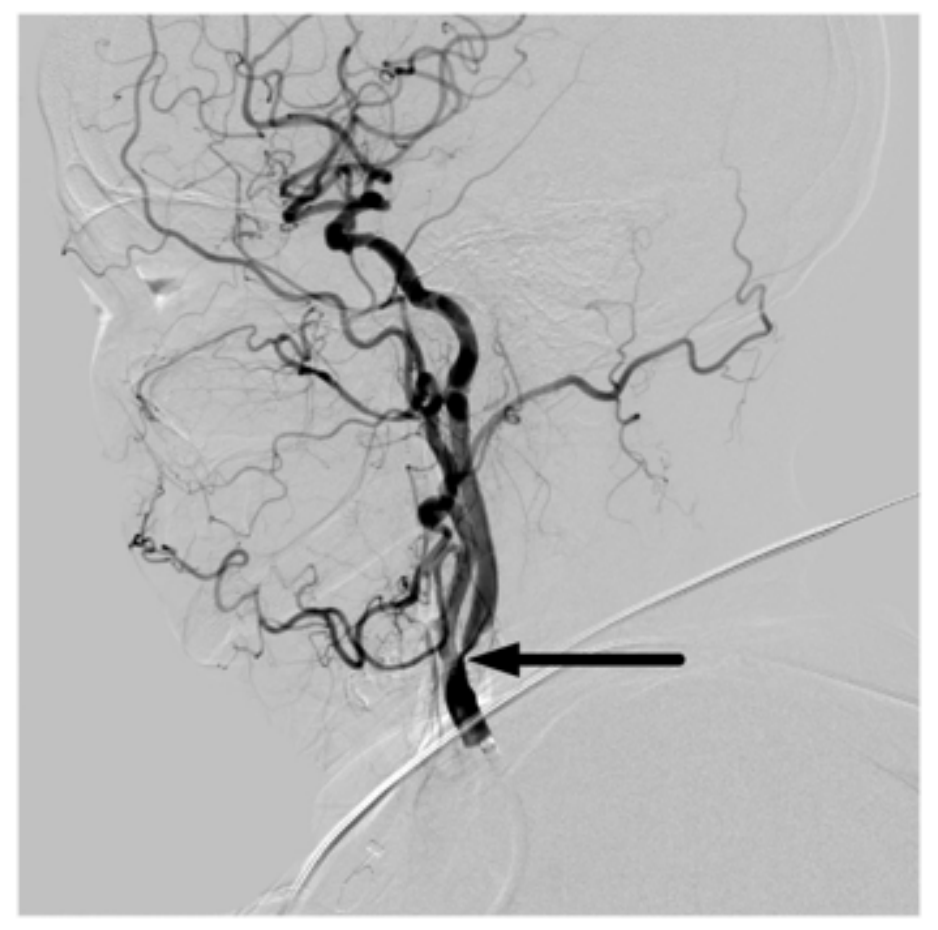

$1 \mathrm{~A}$

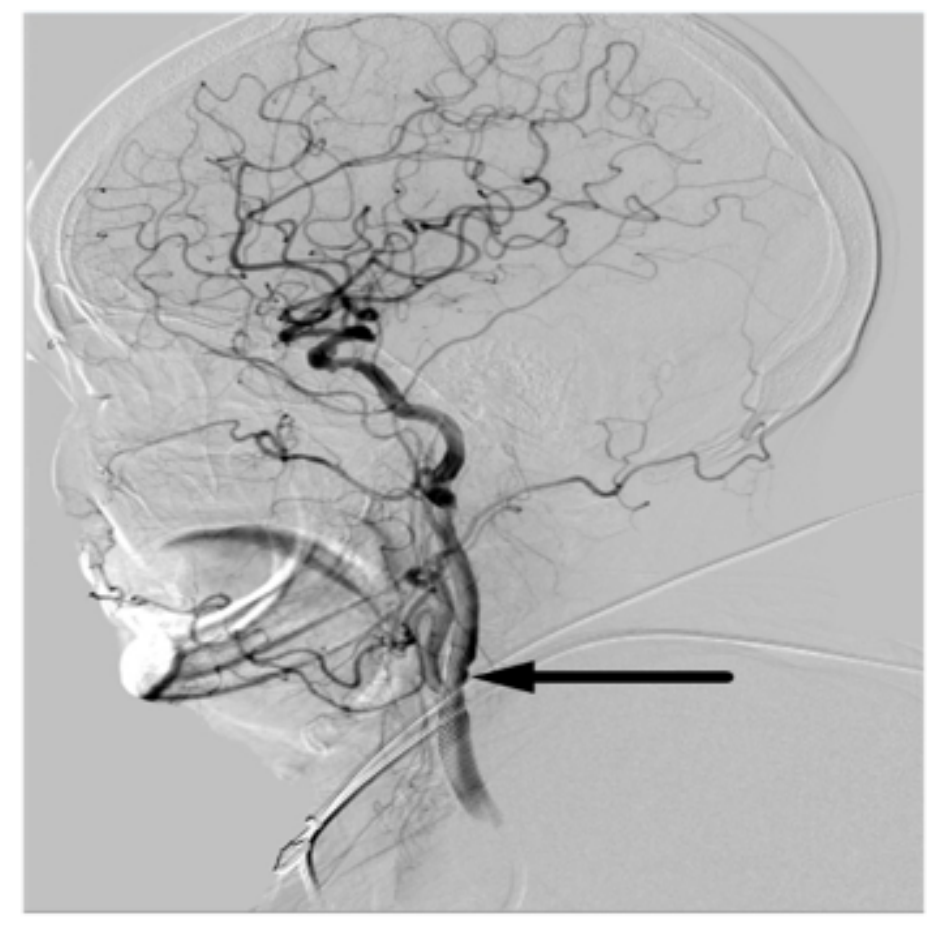

$1 \mathrm{~B}$

\section{Figure 1}

1A: The carotid sinus showed severe stenosis before carotid artery stenting. 1B: The stenosis in carotid sinus was significantly improved after carotid artery stenting and the reperfusion was restored. 


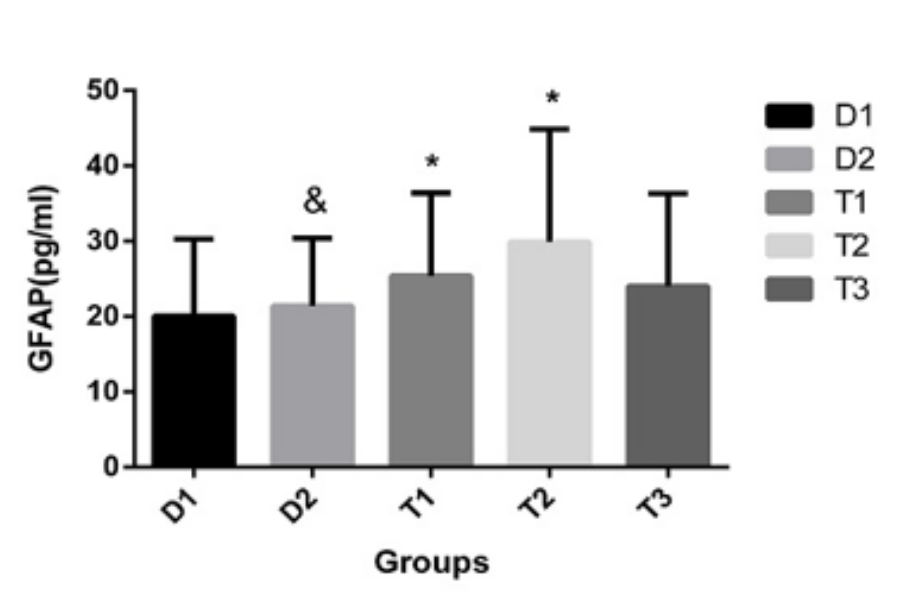

$2 \mathrm{~A}$

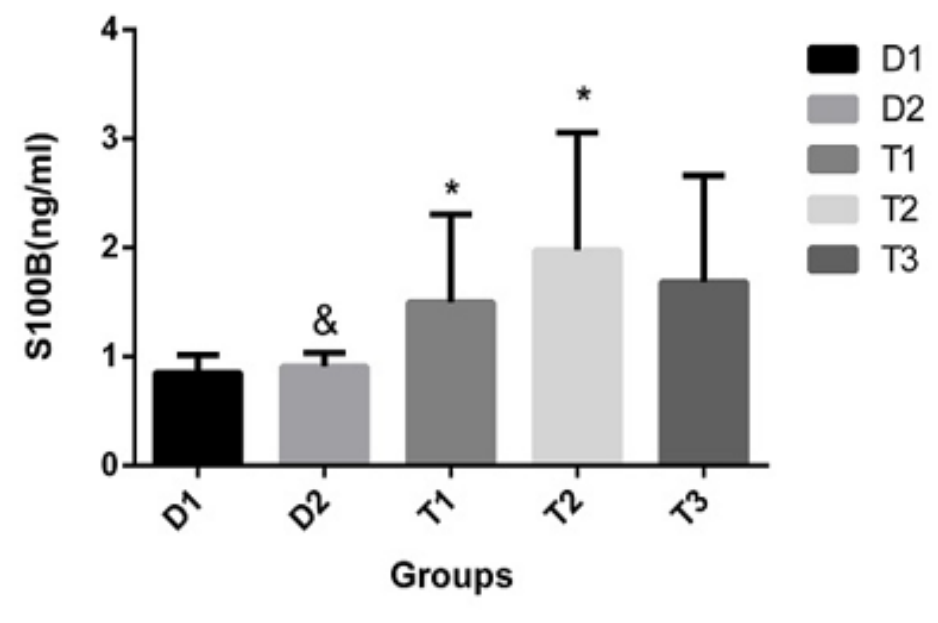

2B

Figure 2

After surgery, the serum concentrations of GFAP (2A) and S100B (2B) in the CAS group increased at T2 and then decreased at $\mathrm{T} 3$, but both proteins in $\mathrm{T} 2$ and $\mathrm{T} 3$ were higher than those at $\mathrm{T} 1 \mathrm{~T} 2>\mathrm{T} 3>\mathrm{T} 1 ; \mathrm{T} 1 \mathrm{vs}$ T2 and T1 vs T3: $P<0.05 区$.

\section{Supplementary Files}

This is a list of supplementary files associated with this preprint. Click to download.

- Titlepage.docx 